\title{
Comparative Therapeutic Efficacy and Safety of Remdesivir monotherapy and its Combination of Lopinavir/Ritonavir in COVID-19 Patients
}

\author{
Marian Boshra ${ }^{1}$, haitham saeed ${ }^{2}$, Ahmed E. Abou Warda ${ }^{3}$, and Rania Sarhan ${ }^{1}$ \\ ${ }^{1}$ Beni Suef University Faculty of Pharmacy \\ ${ }^{2}$ Affiliation not available \\ ${ }^{3}$ October 6 University Faculty of Pharmacy
}

April 19, 2021

\begin{abstract}
Objectives: The treatment of COVID-19 infection remains a challenge because till now, there is no approved therapy for it. This study aimed to estimate the difference in the therapeutic efficacy and safety between remdesivir as monotherapy and its use in combination with lopinavir/ritonavir provided with standard supportive care. Methods: This is a prospective randomized cohort study included 1043 adult patients with confirmed moderate and severe COVID-19 infection. Treatment of all patients followed Egyptian Ministry of Health COVID-19 protocol as the first group received IV remdesivir 200 mg on day 1, followed by $100 \mathrm{mg}$ once daily, for 5 days while the second group received lopinavir/ritonavir 400/100 mg twice daily, for 5 days with the same remdesivir regimen in the first group. All laboratory and clinical parameters were assessed before and after treatment duration. Results: There was no significant difference related to improvement parameters such as laboratory data and improvement time between the two groups. On the other hand, hepatotoxicity of the second group (combination) was significantly higher compared with that of the first one. The elevation on liver enzymes was affected by the severity of the disease, the severe cases showed a high enzyme elevation rate. Conclusion: Remdesivir as monotherapy and its use in combination with lopinavir/ritonavir is effective in the management of moderate COVID 19 subjects than severe cases. The combination of remdesivir with lopinavir/ritonavir is not recommended due to the increased hepatotoxicity effect.
\end{abstract}

Comparative Therapeutic Efficacy and Safety of Remdesivir monotherapy and its Combination of Lopinavir/Ritonavir in COVID-19 Patients

\section{Abstract}

Objectives: The treatment of COVID-19 infection remains a challenge because till now, there is no approved therapy for it. This study aimed to estimate the difference in the therapeutic efficacy and safety between remdesivir as monotherapy and its use in combination with lopinavir/ritonavir provided with standard supportive care.

Methods: This is a prospective randomized cohort study included 1043 adult patients with confirmed moderate and severe COVID-19 infection. Treatment of all patients followed Egyptian Ministry of Health COVID-19 protocol as the first group received IV remdesivir $200 \mathrm{mg}$ on day 1, followed by $100 \mathrm{mg}$ once daily, for 5 days while the second group received lopinavir/ritonavir 400/100 mg twice daily, for 5 days with the same remdesivir regimen in the first group. All laboratory and clinical parameters were assessed before and after treatment duration.

Results: There was no significant difference related to improvement parameters such as laboratory data and improvement time between the two groups. On the other hand, hepatotoxicity of the second group 
(combination) was significantly higher compared with that of the first one. The elevation on liver enzymes was affected by the severity of the disease, the severe cases showed a high enzyme elevation rate.

Conclusion: Remdesivir as monotherapy and its use in combination with lopinavir/ritonavir is effective in the management of moderate COVID 19 subjects than severe cases. The combination of remdesivir with lopinavir/ritonavir is not recommended due to the increased hepatotoxicity effect.

Key words: Remdesivir ; Lopinavir/Ritonavir; COVID 19; Hepatotoxicity

\section{Introduction}

The Coronavirus disease 2019 (COVID-19) is a type of viral pneumonia that is released from severe acute respiratory syndrome coronavirus 2 (SARS-CoV-2). There are very few options for the effective treatment for viral diseases as COVID $19(1,2)$. The presence of different protein targets in certain drugs, in addition to many viral illnesses, leads to overlapping in the molecular paths (3). Therefore, reusing variable drugs for achieving different objectives and determining their effective uses can be a very helpful method in decreasing the time that is required to find new therapy for emergency diseases (4). The widespread clinical and laboratory efficacy and the well-known knowledge about the antiviral mechanisms of remdesivir and lopinavir/ ritonavir combination in the treatment of certain diseases such as the Middle East respiratory syndrome (MERS) and severe acute respiratory syndrome (SARS), was an important cause for using them in novel COVID 19 infection (5). Therefore, a lot of permissions have been given by the World Health Organization (WHO) for examining different drugs in clinical trials to estimate their efficacy and safety against COVID-19 infection $(4,6)$. The administration of drugs alone or in combinations have been tested as remdesivir alone; a combination of lopinavir (LPV) and ritonavir (RTV); a combination of lopinavir, ritonavir, interferon-beta (IFNb); and hydroxychloroquine ( HCQ) (7).

Remdesivir is a broad-spectrum antiviral nucleotide agent. It was used for the treatment of Ebola virus (EBOV) infection in 2017 (8). It has a great antiviral efficacy against a lot of RNA viruses such as the Ebola virus, Hendra virus, MERS-CoV, SARS-CoV, and respiratory syncytial virus (RSV). It is considered a monophosphoramidate prodrug and an adenosine analog (9). In the human body, remdesivir is metabolized by conversion of its prodrug form into its active form, GS-441524, which disorganizes the viral RNA polymerase and evades proofreading by viral exonuclease, leading to a decrease in RNA production. So, the antiviral mechanism of remdesivir can be considered as a delayed chain stopping of nascent viral RNA (10).

Lopinavir (LPV) is a human immunodeficiency virus 1 (HIV-1) protease inhibitor which, in most cases, is used in combination with ritonavir (RTV) to increase the half-life of LPV by the inhibition of cytochrome P4507 (11, 12).

Gastrointestinal side effects of remdesivir and lopinavir/ritonavir combination have been observed during their administration in the treatment of COVID 19 patients especially hepatotoxicity $(13,14)$. However, different heterogeneous cases of liver damage may be present under hepatotoxicity terms with rare symptoms or the appearance of jaundice after 1 to 8 weeks after the initiation of the treatment (15). Consequently, reduction of the treatment dose or discontinuation of these drugs should be done upon the appearance of hepatotoxic effect (16).

Till now, antiviral therapy such as hydroxychloroquine, remdesivir, lopinavir/ritonavir can be considered as drugs under trial and have not been formally approved in the treatment of COVID-19 (17). Therefore, a multifaceted viral target study was recommended to evaluate and compare the therapeutic efficacy and the safety of using Remdesivir monotherapy and the combination of lopinavir /ritonavir and Remdesivir and to determine the mortality rate, the length of stay in the hospital, and the medication effect on clinical and laboratory parameters in patients with moderate and severe COVID-19 infection.

The current study aimed at studying the efficacy and hepatotoxicity of using remdesivir alone and in combination with lopinavir/ritonavir for treatment of COVID-19 adult subjects of moderate to severe cases.

\section{Patients and methods}




\subsection{Study design and participants}

The study was prospective, randomized, cohort study that included 1043 adult patients aged [?] 18 years with confirmed COVID-19 infection between October 1, 2020, and January 30, 2021, from the Hospital of Health Insurance, Beni-Suef, Egypt. These patients were randomized into two groups. The first group of patients $(\mathrm{n}=574)$ received IV remdesivir of $200 \mathrm{mg}$ on day 1 followed by $100 \mathrm{mg}$ once daily infused over 60 minutes for 7 days while the second group of patients $(n=469)$ received lopinavir/ritonavir 400/100 (Kaletra@), twice daily for 7 days with the same remdesivir regimen used in the first group in addition to the standard management.

\subsection{Clinical classification and clinical follow-up}

Egyptian Ministry of Health COVID-19 protocol was used as a guideline for therapeutic management of moderate and severe COVID-19 cases included in the study in addition to the studied antivirals. Both groups were received the same standard management, as well as steroids such as dexamethasone $6 \mathrm{mg} / \mathrm{day}$, was added.

Inclusion criteria included the following: A) Patients with COVID-19 infection who were confirmed with polymerase chain reaction (PCR); B) pulmonary involvement which was detected by oxygen saturation $(\mathrm{Sa02})$ of $<92 \%$ when at ambient air or respiratory rate $(\mathrm{RR})>30$, or $\mathrm{PaO} 2 / \mathrm{FiO} 2$ ratio $<300$; for severe cases or between 92: $95 \%$ for moderate cases. C) Worsening of lung involvement defined as an increase in the number and /or extension of pulmonary areas of consolidation, need for increased FiO2 to maintain stable $\mathrm{O} 2$ saturation or worsening $\mathrm{O} 2$ saturation of $>3 \%$ with stable $\mathrm{FiO} 2$.

Exclusion criteria included A) baseline evaluation of alanine aminotransferase (ALT) or aspartate aminotransferase (AST) levels $>3: 5$ fold the upper limit of the normal range and estimated creatinine clearance less than $30 \mathrm{ml} / \mathrm{min}$; B) Pregnancy; C) Known hypersensitivity to drugs or any component of the formulation, D) Serious co-morbidities as hepatic patients Child-Pugh class C.

\subsection{Study population}

The following baseline features were analyzed: age, sex, duration of symptoms, comorbidities (smoking history, chronic kidney disease, arterial hypertension, cancer, type 2 diabetes mellitus, coronary artery disease, and chronic obstructive pulmonary disease), baseline clinical status (need for supplementary oxygen therapy, need for invasive or noninvasive ventilation, body temperature), chest computed tomographic (CT) scan and serum inflammatory markers (CRP, ferritin, LDH).

\subsection{Clinical outcomes}

\subsubsection{Primary outcomes:}

The proportion of clinically improved patients in the interventional group compared with the proportion of improved patients in the other group.

Patients' clinical improvement (recovery) was assessed based on improvement in oxygenation or patient discharge, and death.

Time to clinical improvement.

Mortality rate.

\subsubsection{Secondary outcomes}

Monitoring of adverse events: the occurrences of adverse events were recorded daily, with a focus on the elevation of AST or ALT level > 3x the upper limit of the normal range.

\subsection{Chest computed tomography}


Chest computed tomographic (CT) scans were carried out for all patients with COVID 19 infection and analyzed. The patients with severe COVID 19 infection had chest radiology showing more than $50 \%$ lesion or progressive lesion.

\subsection{Statistical analysis}

Data has been analyzed using SPSS version 25.0 (SPSS, Chicago, IL-USA). Continuous variables were reported as mean $\pm \mathrm{SD}$. Categorical variables were reported as numbers and percentages. T-test tests were applied to continuous variables and Chi-square and Mcnemar tests were used for categorical variables. Pvalues $<0.05$ were considered statistically significant. Response surface plots were produced by introducing input variables and output responses that were encoded numerically using design expert $\mathrm{v} 7$.

\subsection{Ethics statement}

The study was carried out after approval from the Research Ethical Committee of Faculty of Pharmacy, BeniSuef University with a serial number (REC-H-PhBSU-21001). Informed written consent was taken from each study subject and they were informed that their participation is voluntary, and they can withdraw from the study at any time. The study was performed according to the good clinical practices recommended by the Declaration of Helsinki and its amendments.

\section{Results}

About 1043 adult COVID-19 subjects were recruited in the study; they were divided into 2 groups their ages, expressed as mean $\pm \mathrm{SD}$, were $64.45 \pm 12.01$ and $60.18 \pm 12.09$ respectively for the first and second group. The first group included 574 (238 females) patients, while the second group included 469 (154 females). The two groups included 336 and 343 severe cases respectively and the rest of the cases were moderate (Table 1). Laboratory data were expressed in Table 2.

Regarding the effect of both interventions on the efficacy of treatment, the significance of each intervention was as follows; the mortality rate for remdesivir group was $21.95 \%$ which is not significantly different from that $22.39 \%$ of the remdesivir plus Kaletra $($ group.

Days till recovery of subjects were variable (5-15 days) between subjects of the same group but the mode was 7 days for both groups which reflected a non-significant effect of both treatments on the recovery days. Regarding the effect of other factors on the recovery days, the severity of the disease for remdesivir group showed a significant $(\mathrm{P}<0.001)$ effect on the number of days required for patient clinical improvement. Also, a significant $(\mathrm{P}<0.01)$ difference was observed regarding the second group and this difference was reflected by fewer recovery days for those with moderate severity compared with more days required for the severe cases. On the other hand, the relation between severity of the case and mortality rate was not the same for both groups. The second group has a significant $(\mathrm{P}<0.01)$ effect of case severity on the rate of mortality while the first group showed no significant difference.

The difference between the number of patients with abnormal laboratory values at baseline and after treatment was significant for most of the items but few items were not significant for both groups. D. Dimer test showed a non-significant difference for both groups. Also, the ALT test had a non-significant increase for remdesivir group only while it was significant $(\mathrm{P}<0.001)$ for the second group. For the second liver-related test, AST was significantly ( $\mathrm{P}=0.016$ for the first group and $\mathrm{P}<0.001$ for the second group) higher after initiation of treatment for both groups. The magnitude of ALT and AST enzymes elevation expressed as folds showed that $203(43.28 \%)$ of the recruited subjects showed more than 3 folds increase in ALT enzyme among subjects of the second group, while only $14(2.44 \%)$ of the first group only had ALT levels elevated more than 3 folds. The same observation was related to AST levels, as 112 (23.88\%) of the second group, subjects showed more than three folds increase in enzyme level, while the first group had no case with this elevation magnitude. Regarding LDH, ferritin, ALC, and CRP, all were improved after the initiation of treatment for both groups (Fig 1).

As shown in fig 2 and fig 3 , response surface plots reflected the effect of treatment options and other variables 
(age, gender, severity, and comorbidities) on mortality rate, and the response surface plots reflected the effect of different variables on mortality rate was shown in fig 2 .

Fig 2 A showed the effect of comorbidity numbers on mortality, it was clear that the higher the number of comorbidities for the subject, the higher the mortality rate. Subjects suffering from cardiovascular, diabetes, and respiratory diseases were among the severe subjects (encoded numerically $=2$ ). Fig 2 B indicated the negative effect of increasing the subject's age on mortality rate, the older the patient the higher the mortality rate. Fig $2 \mathrm{C}$ reflected the effect of disease severity on mortality for both groups, the severe cases had a higher mortality rate compared to moderate cases, and this was consistent with the effect of the comorbidities. Fig $2 \mathrm{D}$ presented the effect of gender on mortality, for remdesivir group there was no difference between males and females, while for the second group it was noted that females have a higher mortality rate compared with males. Effect of treatment option, gender, and severity of the disease on liver enzymes was expressed in response surface plot (Fig 3); adding lopinavir/ritonavir to remdesivir resulted in a significant increase in liver enzymes in a short time. This increase was higher in females compared to males (Fig $3 \mathrm{~A}$ and B) and in severe cases compared with moderate (Fig $3 \mathrm{C}$ and D).

For the second group, ALT and AST levels increased above 3 folds from the baseline for $203(43.2 \%)$ and $112(23.88 \%)$ respectively, with about $4.48 \%$ of subjects with increased levels above 8 folds. This effect was higher for females compared to males (Fig 3) for both ALT and AST; also the mortality rate was higher for females. Regarding the effect of age on mortality rate, subjects less than 40 years old had a lower mortality rate compared with older patients (Fig 2 B).

\section{Discussion}

COVID-19 treatment protocols differ from one country to another and many clinical trials are initiated around the world trying to indicate the beneficial effect of different antiviral therapies. Findings of clinical trials were controversial regarding the beneficial or non-beneficial role of certain antiviral mediations such as remdesivir and a combination of lopinavir/ritonavir drugs (18-21). Remdesivir has been reported to be clinically effective compared with placebo regarding the time needed for COVID-19 patient recovery. However, adverse effects of remdesivir were also reported in the same study for about $24.6 \%$ of recruited subjects (22). Studies carried on lopinavir/ritonavir showed a little benefit for improving the clinical outcome of subjects hospitalized with mild and moderate COVID-19 (23). Hence the current study aimed to evaluate the effect and hepatotoxicity of combining remdesivir with lopinavir/ritonavir.

Using lopinavir/ritonavir alone was reported to be less effective for the management of COVID-19 (24) while combining these antiviral drugs with interferon beta $1 \mathrm{~b}$ has been reported to be safe and more effective compared with lopinavir/ritonavir alone in reducing the duration of coronavirus shedding, improving symptoms, and reducing hospital stay in subjects with mild to moderate cases (25). Also, Verdugo-Paiva et al, indicated that using lopinavir/ritonavir might play a role in improving outcomes of critical and severe cases of COVID-19 (26). Evidence of low certainty suggests that lopinavir/ritonavir may reduce mortality, but as the extent of the reduction differs among various risk groups, there should also be differences in any treatment decision. The evidence also shows that lopinavir/ritonavir can reduce the risk of developing or needing intrusive mechanical ventilation, but the certainty of developing respiratory failure, acute respiratory distress syndrome were reported with these studies (26-28). Hence, combining these antiviral drugs with remdesivir was proposed to enhance the later efficacy and improve COVID-19 patients' outcomes.

The finding of Beigel et al was consistent with findings related to remdesivir efficacy expressed by the percentage $(76.83 \%$ ) of the clinically improved subjects. Previous clinical trials studied the effect of duration therapy and showed that 5 days remdesivir treatment is more beneficial compared with 10 days of therapy (29). One of the most critically reported adverse events was acute hepatotoxicity which has been presented as a dramatic increase in liver enzymes 5 days after initiation of remdesivir therapy (30). However, the use of remdesivir in the current study did not result in a dramatic increase in liver enzymes which was confirmed by the absence of subjects that have elevated AST level 3 folds or more. These findings are consistent with the conclusion of a meta-analysis carried by Yiting et al. (31). There was no significant difference between the two 
groups regarding the improvement of laboratory data reflecting the non-beneficial role for lopinavir/ritonavir. While the significant effect of combination groups was related to hepatotoxicity. Hence, combining lopinavir/ ritonavir with remdesivir is not recommended due to dual causes related to lack of efficacy and increased hepatotoxicity.

\section{Conclusion}

Our findings indicated that remdesivir as monotherapy and its use in combination with lopinavir/ritonavir has a significant difference which was reflected by fewer recovery days for moderate COVID 19 subjects than severe ones. Treatment with remdesivir as a single drug seems to be more safe and effective in reducing mortality rate than its use in combination with lopinavir/ ritonavir. Also, the combination has a high hepatotoxic effect as elevated AST level 3 folds or more.

\section{Acknowledgments}

This research did not receive any specific grant from funding agencies in the public, commercial, or not-forprofit sectors.

\section{Author contributions}

Marian S. Boshra : Conceptualization, Methodology, Writing- Original draft preparation, Investigation,Writing- Reviewing and Editing; Haitham Saeed : Data curation, Software, Writing- Original draft preparation; Ahmed E. Abou Warda : Visualization, Supervision; Rania M Sarhan: Conceptualization, Methodology, Writing- Original draft preparation, Investigation,Writing- Reviewing and Editing

\section{References}

1. Sheahan TP, Sims AC, Leist SR, Schäfer A, Won J, Brown AJ, et al. Comparative therapeutic efficacy of remdesivir and combination lopinavir, ritonavir, and interferon beta against MERS-CoV. Nature communications. 2020;11(1):1-14.

2. Sarhan RM, Madney YM, Abou Warda AE, Boshra MS. Therapeutic efficacy, mechanical ventilation, length of hospital stay, and mortality rate in severe COVID-19 patients treated with tocilizumab. International journal of clinical practice. 2021:e14079.

3. Hendaus MA. Remdesivir in the treatment of Coronavirus Disease 2019 (COVID-19): A simplified summary. Journal of Biomolecular Structure and Dynamics. 2020:1-6.

4. Wu R, Wang L, Kuo H-CD, Shannar A, Peter R, Chou PJ, et al. An update on current therapeutic drugs treating COVID-19. Current Pharmacology Reports. 2020:1.

5. Chan JF, Chan K-H, Kao RY, To KK, Zheng B-J, Li CP, et al. Broad-spectrum antivirals for the emerging Middle East respiratory syndrome coronavirus. Journal of Infection. 2013;67(6):606-16.

6. De Wilde AH, Jochmans D, Posthuma CC, Zevenhoven-Dobbe JC, Van Nieuwkoop S, Bestebroer TM, et al. Screening of an FDA-approved compound library identifies four small-molecule inhibitors of Middle East respiratory syndrome coronavirus replication in cell culture. Antimicrobial agents and chemotherapy. 2014;58(8):4875-84.

7. Maciorowski D, Idrissi SZE, Gupta Y, Medernach BJ, Burns MB, Becker DP, et al. A review of the preclinical and clinical efficacy of remdesivir, hydroxychloroquine, and lopinavir-ritonavir treatments against COVID-19. SLAS DISCOVERY: Advancing the Science of Drug Discovery. 2020;25(10):1108-22.

8. Beigel JH, Tomashek KM, Dodd LE, Mehta AK, Zingman BS, Kalil AC, et al. Remdesivir for the treatment of Covid-19 - preliminary report. The New England journal of medicine. 2020.

9. Al-Tawfiq JA, Al-Homoud AH, Memish ZA. Remdesivir as a possible therapeutic option for the COVID19. Travel medicine and infectious disease. 2020. 
10. Gordon CJ, Tchesnokov EP, Feng JY, Porter DP, Gotte M. The antiviral compound remdesivir potently inhibits RNA-dependent RNA polymerase from Middle East respiratory syndrome coronavirus. Journal of Biological Chemistry. 2020;295(15):4773-9.

11. Uzunova K, Filipova E, Pavlova V, Vekov T. Insights into antiviral mechanisms of remdesivir, lopinavir/ritonavir and chloroquine/hydroxychloroquine affecting the new SARS-CoV-2. Biomed Pharmacother. 2020:110668.

12. Alanagreh La, Alzoughool F, Atoum M. The human coronavirus disease COVID-19: its origin, characteristics, and insights into potential drugs and its mechanisms. Pathogens. 2020;9(5):331.

13. Fu B, Xu X, Wei H. Why tocilizumab could be an effective treatment for severe COVID-19? Journal of translational medicine. 2020;18(1):1-5.

14. Ghosh CK, Hasan SA, Dey S. Remdesivir Induced Liver Injury and Severe COVID-19 Infection. American Journal of Internal Medicine. 2020;8(6):285-8.

15. Canta F, Marrone R, Bonora S, D'Avolio A, Sciandra M, Sinicco A, et al. Pharmacokinetics and hepatotoxicity of lopinavir/ritonavir in non-cirrhotic HIV and hepatitis C virus (HCV) co-infected patients. Journal of Antimicrobial Chemotherapy. 2005;55(2):280-1.

16. Arabi YM, Alothman A, Balkhy HH, Al-Dawood A, AlJohani S, Al Harbi S, et al. Treatment of Middle East respiratory syndrome with a combination of lopinavir-ritonavir and interferon- $\beta 1 \mathrm{~b}$ (MIRACLE trial): study protocol for a randomized controlled trial. Trials. 2018;19(1):1-13.

17. Little P. Non-steroidal anti-inflammatory drugs and covid-19. British Medical Journal Publishing Group; 2020 .

18. Cao B, Wang Y, Wen D, Liu W, Wang J, Fan G, et al. A trial of lopinavir-ritonavir in adults hospitalized with severe Covid-19. 2020.

19. Owa AB, Owa OTJJoM, Immunology, Infection. Lopinavir/ritonavir use in Covid-19 infection: is it completely non-beneficial? 2020;53(5):674-5.

20. Horby PW, Mafham M, Bell JL, Linsell L, Staplin N, Emberson J, et al. Lopinavir-ritonavir in patients admitted to hospital with COVID-19 (RECOVERY): a randomised, controlled, open-label, platform trial. 2020;396(10259):1345-52.

21. Wang Y, Zhang D, Du G, Du R, Zhao J, Jin Y, et al. Remdesivir in adults with severe COVID-19: a randomised, double-blind, placebo-controlled, multicentre trial. 2020;395(10236):1569-78.

22. Madsen LWJTNEJoM. Remdesivir for the Treatment of Covid-19-Final Report. 2020;338(19):1813-26.

23. Li Y, Xie Z, Lin W, Cai W, Wen C, Guan Y, et al. Efficacy and safety of lopinavir/ritonavir or arbidol in adult patients with mild/moderate COVID-19: an exploratory randomized controlled trial. 2020;1(1):105-13. $\mathrm{e} 4$.

24. Guan W-j, Ni Z-y, Hu Y, Liang W-h, Ou C-q, He J-x, et al. Clinical characteristics of coronavirus disease 2019 in China. 2020;382(18):1708-20.

25. Hung IF-N, Lung K-C, Tso EY-K, Liu R, Chung TW-H, Chu M-Y, et al. Triple combination of interferon beta-1b, lopinavir-ritonavir, and ribavirin in the treatment of patients admitted to hospital with COVID-19: an open-label, randomised, phase 2 trial. 2020;395(10238):1695-704.

26. Verdugo-Paiva F, Izcovich A, Ragusa M, Rada G, Group C-LOW. Lopinavir/ritonavir for COVID-19: a living systematic review. Medwave. 2020;20(6).

27. Verdugo-Paiva F, Izcovich A, Ragusa M, Rada G, Medwave C-LOWGJ. Lopinavir/ritonavir for COVID19: a living systematic review. 2020;20(6). 
28. Horby PW, Mafham M, Bell JL, Linsell L, Staplin N, Emberson J, et al. Lopinavir-ritonavir in patients admitted to hospital with COVID-19 (RECOVERY): a randomised, controlled, open-label, platform trial. The Lancet. 2020;396(10259):1345-52.

29. Jiang Y, Chen D, Cai D, Yi Y, Jiang SJJomv. Effectiveness of remdesivir for the treatment of hospitalized COVID-19 persons: A network meta-analysis. 2021;93(2):1171-4.

30. Leegwater E, Strik A, Wilms EB, Bosma LB, Burger DM, Ottens TH, et al. Drug-induced liver injury in a COVID-19 patient: potential interaction of remdesivir with P-glycoprotein inhibitors. 2020.

31. Li Y, Cai H, Rajabalee N, Au X, Friedenberg F, Wallach SJOjotACoG, et al. S1027 Hepatotoxicity of Remdesivir for COVID-19: Systematic Review and Meta-Analysis. 2020;115:S523.

Table 1 . Demographic data and medical characteristics at baseline.

\begin{tabular}{lll}
\hline & Group 1 (Remdesivir) & Group 2 (Remdesivir + Kaletra) \\
\hline Age (mean \pm SD) & $64.45 \pm 12.01$ & $60.18 \pm 12.09$ \\
Gender (n) & 336 males, 238 females & 315 males, 154 females \\
Severity (n) & 336 severe, 238 moderate & 343 severe, 126 moderate \\
Oxygen saturation (mean \pm SD) & $86.43 \pm 11.79$ & $84 \pm 11$ \\
Max Temperature & $38.14 \pm 0.88$ & $38.09 \pm 0.69$ \\
Recovered cases (n) & $448(78.05 \%)$ & $364(77.61 \%)$ \\
Dead cases (n) & $126(21.95 \%)$ & $105(22.39 \%)$ \\
Comorbidities & Comorbidities & Comorbidities \\
Hypertension n (\%) & $287(50 \%)$ & $252(53.3 \%)$ \\
Diabetes n (\%) & $245(42.68 \%)$ & $182(38.81 \%)$ \\
Chronic kidney disease n (\%) & $28(4.88 \%)$ & - \\
Chronic liver disease n (\%) & $21(3.66 \%)$ & $35(7.46 \%)$ \\
Ischemic heart disease n (\%) & $140(24.39 \%)$ & $14(2.98 \%)$ \\
Atrial fibrillation n (\%) & $63(10.98 \%)$ & - \\
COPD n (\%) & $35(6.1 \%)$ & - \\
Asthma n (\%) & $14(2.44 \%)$ & $14(2.98 \%)$ \\
Obesity n (\%) & $14(2.44 \%)$ & - \\
Ventilation & Ventilation & Ventilation \\
low supplementary oxygen n (\%) & $308(53.66 \%)$ & $175(37.31 \%)$ \\
Non-invasive ventilation n (\%) & $231(40.24 \%)$ & $287(61.19 \%)$ \\
Invasive ventilation n (\%) & $35(6.1 \%)$ & $21(20.1 \%)$ \\
ICU admission n (\%) & $329(57.32 \%)$ & $343(73.13 \%)$ \\
\hline
\end{tabular}

Table 2. Number (\%) of subjects with abnormal Laboratory data for both groups at baseline and after end of therapy.

\begin{tabular}{lllllllll}
\hline & & $\begin{array}{l}\text { D- } \\
\text { Dimer }\end{array}$ & LDH & Ferritin & CRP & ALC & ALT & AST \\
\hline Group 1 & Remdisivir & 224 & 378 & 406 & 553 & 511 & 147 & 280 \\
& (basline) & $(39.02 \%)$ & $(65.85 \%)$ & $(70.73 \%)$ & $(96.34 \%)$ & $(89.02 \%)$ & $(25.61 \%)$ & $(48.78 \%$ \\
& Remdisivir & 217 & 364 & 315 & 441 & 448 & 224 & 364 \\
& (after) & $(37.8 \%)$ & $(63.41 \%)$ & $(54.88 \%)$ & $(76.83 \%)$ & $(78.05 \%)$ & $(39.02 \%)$ & $(63.41 \%$ \\
Group 2 & Remdisivir & 280 & 378 & 350 & 455 & 406 & 133 & 210 \\
& + Kaletra & $(59.7 \%)$ & $(80.6 \%)$ & $(74.63 \%)$ & $(97.01 \%)$ & $(86.57 \%)$ & $(28.36 \%)$ & $(44.78 \%$ \\
& (basline) & & & & & & &
\end{tabular}




\begin{tabular}{llllllll}
\hline & D- & & & & & \\
& Dimer & LDH & Ferritin & CRP & ALC & ALT & AST \\
\hline Remdisivir & 231 & 308 & 378 & 378 & 287 & 427 & 378 \\
$\begin{array}{l}\text { + Kaletra } \\
\text { (after) }\end{array}$ & $(49.25 \%)$ & $(65.67 \%)$ & $(80.6 \%)$ & $(80.6 \%)$ & $(61.19 \%)$ & $(91.04 \%)$ & $(80.6 \%)$ \\
\hline
\end{tabular}

\section{Figure legend}

Figure 1. The number of subjects with abnormal laboratory data values at baseline and after treatment.

Figure 2. Response surface plot reflect the effect of different variables, A) comorbidities, B) age, C) disease severity, and D) subject gender on the mortality rate of both groups.

Figure 3. Response surface plot reflects the effect of different variables, A and B) Gender, and C and D) disease severity on liver enzyme elevations for both groups.

\section{Hosted file}

Figures.pdf available at https://authorea.com/users/396160/articles/518640-comparativetherapeutic-efficacy-and-safety-of-remdesivir-monotherapy-and-its-combination-oflopinavir-ritonavir-in-covid-19-patients 